\title{
SPARSE ACTUATOR ARRAY COMBINED WITH INVERSE FILTER FOR MULTITOUCH VIBROTACTILE STIMULATION.
}

\author{
Lucie Pantera ${ }^{1}$ and Charles Hudin ${ }^{1}$
}

\begin{abstract}
Because vibrations propagate and reverberate, providing precise and distinct vibrotactile stimulation to multiple fingers in contact simultaneously with a touch surface is challenging. Previous research has demonstrated that it is possible to render localised multitouch feedback using piezoelectric actuators. This paper shows that a sparse array of piezoelectric actuators glued to the surface and driven by an inverse filtered signal can overcome those propagation effects and produce undistorted and independent vibrotactile stimuli. Different experiments have been conducted in order to prove that it is possible to realise localised multitouch in any point of a plate. Then an experiment have been realised in order to highlight the impact of the finger on the stimulation signal.
\end{abstract}

\section{INTRODUCTION}

The benefits of providing a vibrotactile feedback during the interaction with a touch sensitive surface are numerous and well established. Pleasantness of the interaction, rapidity of task completion, reduction of cognitive load are often cited [1], [2], [3], [4]. Besides action confirmation, vibrations combined with force sensing and synthesis algorithm can raise the illusion of textured and compliant surfaces [5], [6], [7], thus engaging users into more immersive interactions. Standard approaches, as they vibrate the entire interaction surface cannot provide distinct stimulations to multiple fingers in contact. The added value of those approaches in the case of multitouch or multi user interactions is thus greatly reduced.

Controlling the vibration field over the entire interaction surface is one way to stimulate independently multiple fingers. Several techniques, based on wave interference have been proposed. The time reversal approach focuses bending waves to produce localized impulsive displacements on transparent glass plates [8]. Phased actuator array can focus ultrasounds to create localized mid-air sensations [9]. Modal synthesis combines vibration modes of the surface to vibrate chosen locations while cancelling others [10], [11]. All those techniques operate remotely from actuators, in their far field where diffraction limits the resolution to the wavelength scale. To reach small wavelength and obtain spatial resolutions compatible with finger or hand dimensions, those techniques operate at ultrasonic frequencies, far beyond the tactile sensitivity range. The perception of such stimulation then relies on nonlinear demodulation phenomena (acoustic radiation pressure, intermittent contact or squeeze film effect) that are not easily controlled. On the other hand, low frequency implementations of those approaches always yield

\footnotetext{
${ }^{1}$ Lucie Pantera and Charles Hudin are from CEA, LIST, F91191 Gif-sur-Yvette, FRANCE. lucie.pantera@cea.fr charles.hudin@cea.fr
}

to poorly localized stimulations [12], [13], [14].

Inverse filter is an other technique that finds applications in medical imaging [15] and spatial audio rendering [16], [17] but suffers from the same diffraction limit. In those cases the difficulty, from the far field, to control or distinguish points much closer than the wavelength appears as an ill condition of the propagation matrix to be inverted. The resolution in medical imaging applications is thus limited to half the wavelength and crosstalk cancellation in audio is known to be unstable at low frequency. An extreme case of near field operation consists in collocating control points and actuators. It has been demonstrated that the inverse filter approach is very effective in this configuration to control the vibrotactile stimuli provided to multiple fingers, despite their small distance with respect to the wavelength.

We show in this paper that vibrotactile stimuli can also be controlled effectively at positions close to the actuators, at distances small in comparison to their size. A set of actuators, arranged as a sparse array spanning the entire surface without necessarily covering surface was thus employed. With this implementation, vibrotactile stimuli can be delivered to fingers at arbitrary positions on the plate and not only above actuators. But this array of actuators has limitations. Indeed it covers the entire surface of the glass plate and actuators are not yet transparent, this surface cannot be stacked over a screen. Then, fingers positions must be known at all time to update filters and calculate the driving signals. A touch sensitive surface is therefore required. Moreover the inverse filter method requires many calculation steps (inversion of matrix, updating the position of the fingers, signal filtering) and must be optimised to operate in real time. Lastly, this technique needs a calibration step whose stability in time has not been evaluated yet. Nevertheless, we have a good resolution: around $1.5 \mathrm{~cm}$ far below the wavelength $\lambda_{250 \mathrm{~Hz}}=19 \mathrm{~cm}$. The signals have frequencies bellow $1 \mathrm{kHz}$, which is preferable for the perception and the power consumption. Finally the prototype is easy to reproduce. It has been realised with inexpensive piezoelectric components, miniaturized off-the-shelf piezoelectric drivers and standard capacitive screen. We successfully controlled the vibration of up to three points with various frequencies within tactile sensitivity range. Finally, the influence of finger contact and pressure on the effectiveness of the inverse filter is assessed in a preliminary study.

\section{INVERSE FILTER PRINCIPLE}

The inverse filter approach relies on a set of actuators to control the out of plane displacement at a set of finger 
positions on the touch panel. The following equation captures the relationship between a signal $s_{i}(t)$ driving an actuator $i$ and the displacement $u_{j}(t)$ at position $j$ :

$$
u_{j}(t)=h_{j i}(t) \otimes s_{i}(t)
$$

where $\otimes$ is the convolution operator and $h_{j i}(t)$ the impulse response between actuator $i$ and point $j$. In the frequency domain, this equation writes

$$
U_{j}(\omega)=H_{j i}(\omega) . S_{i}(\omega)
$$

where $\omega$ is the circular frequency and $H_{j i}$ the transfer function. In our experiment, we consider the general case of $\mathrm{N}$ actuators and $\mathrm{M}$ control points. Because the system is considered as linear, the displacement at each control point is the addition of all actuators contribution, thus leading to a matrix relation between the sets of displacements and driving signals:

$$
\left(\begin{array}{c}
U_{1} \\
U_{2} \\
\vdots \\
U_{M}
\end{array}\right)=\left(\begin{array}{cccc}
H_{11} & H_{12} & \ldots & H_{1 N} \\
H_{21} & H_{22} & \ldots & H_{2 N} \\
\vdots & \vdots & \ddots & \vdots \\
H_{M 1} & H_{M 2} & \ldots & H_{M N}
\end{array}\right)\left(\begin{array}{c}
S_{1} \\
S_{2} \\
\vdots \\
S_{N}
\end{array}\right)
$$

In condensed form, this matrix equation writes:

$$
\mathbf{U}(\omega)=\mathbf{H}(\omega) \mathbf{S}(\omega)
$$

The idea of inverse filtering is to inverse, for each frequency components, the $\mathbf{H}(\omega)$ matrix in order to calculate the driving signals $\mathbf{S}(\omega)$ that will produce the target displacements $\mathbf{U}(\omega)$ at the set of control points. Since, in our case the number of control points and actuators are different, the inverse operation is achieved by a pseudo-inverse based on a singular-value decomposition instead of a classical matrix inversion. The driving signal $\mathbf{S}(\omega)$ is therefore calculated as follows:

$$
\mathbf{S}=\mathbf{H}^{+} \mathbf{U}
$$

where $\mathbf{H}^{+}$is the pseudo-inverse. Finally, the signal $\mathbf{S}$ is transformed back in the time domain by an Inverse Fourier Transform to drive the actuators.

\section{EXPERIMENTAL VALIDATION}

\section{A. Setup}

The different experiments were carried out on the setup depicted in Fig. 1. Fourteen piezoelectric actuators (piezoelectric diaphragms 7BB Murata, $20 \mathrm{~mm}$ diameter) were glued on the bottom of a $96 \times 162 \times 1 \mathrm{~mm}$ touchscreen (7" pingbo PB70DR8272-R1) as shown in Fig. 1. This screen was glued on a $3 \mathrm{~d}$ printed plastic frame with the same external dimensions. Both were screwed on a rigid plastic plate. Actuators were driven individually with piezo haptic drivers (DRV8662-Texas Instrument) delivering up to 200 Vpp. Surface displacements were measured by a laser vibrometer (Polytec OFV-534/2570) mounted on a motorised

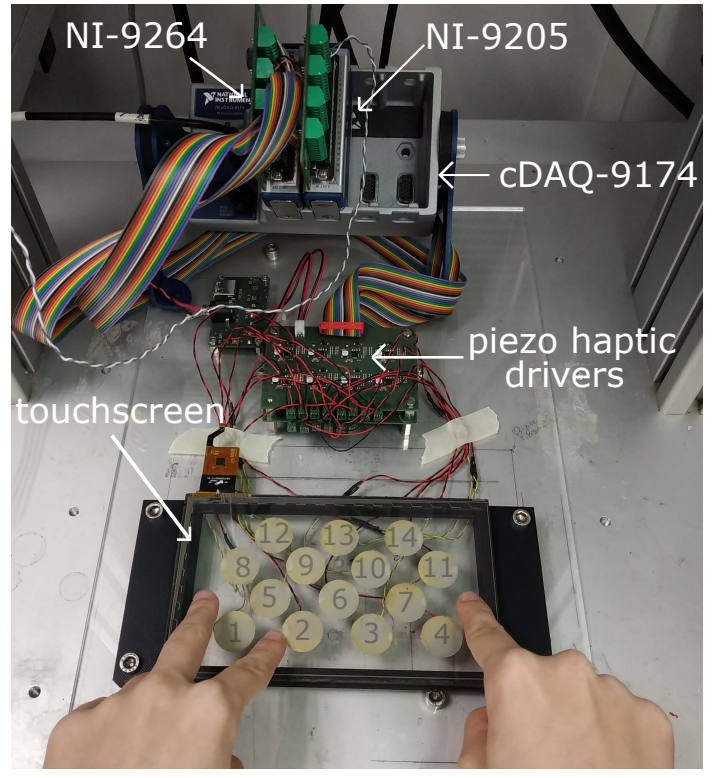

Fig. 1. Electronic interface of the prototype: acquisition device NI-9264 and NI-9205, electronic support cDAQ-9174, piezo haptic drivers which drive piezoelectric actuators. The touchscreen where is glued the fourteen piezoelectric actuators.

three axis platform. The acquisition device (NI-9264, NI9205 and cDAQ-9174) (see Fig. 1) allowed for a synchronous emission and acquisition of actuators and vibrometer signals. All signals were sampled at $F_{s}=20 \mathrm{kHz}$.

\section{B. System calibration}

The matrix $\mathbf{H}$ captures the mechanical transduction of the actuators as well as the propagation, reverberation and attenuation of waves into the touch surface. Instead of simulating all those phenomena, we chose to acquire experimentally this matrix. Each entry $H_{j i}$ was measured for all frequencies at once with the following method:

We sent a swept sine signal of duration $T=200 \mathrm{~ms}$, with frequency varying linearly from 0 to $F_{s} / 2=10 \mathrm{kHz}$ to actuator $i$ and measured synchronously the resulting displacement, containing $N_{\text {sample }}=F_{s} \times T$ samples, at control point $j$. A Fourier Transform was performed on those two signals. The transfer function $H_{j i}$ was calculated as the ratio, in the frequency domain, of the displacement by the driving signal. The same procedure was repeated for all actuators and all control points. Fig. 2 summarize this procedure.

\section{Control on two positions}

The first set of experiment consisted in controlling the vibrations at two random positions on the surface. Vibrotactile stimuli were set as target vibration $\mathbf{u}(t)$. Those vibrotactile signals were either a zero displacement or a six cycles burst sine with different characteristics:

\begin{tabular}{|c||c|c|c|c|}
\hline Frequency $(\mathrm{Hz})$ & 250 & 300 & 500 & 700 \\
\hline Duration $(\mathrm{ms})$ & 24 & 20 & 12 & 8 \\
\hline
\end{tabular}

This burst is modulated by a hamming window. The choice of the frequencies is arbitrary but we wanted to cover the 


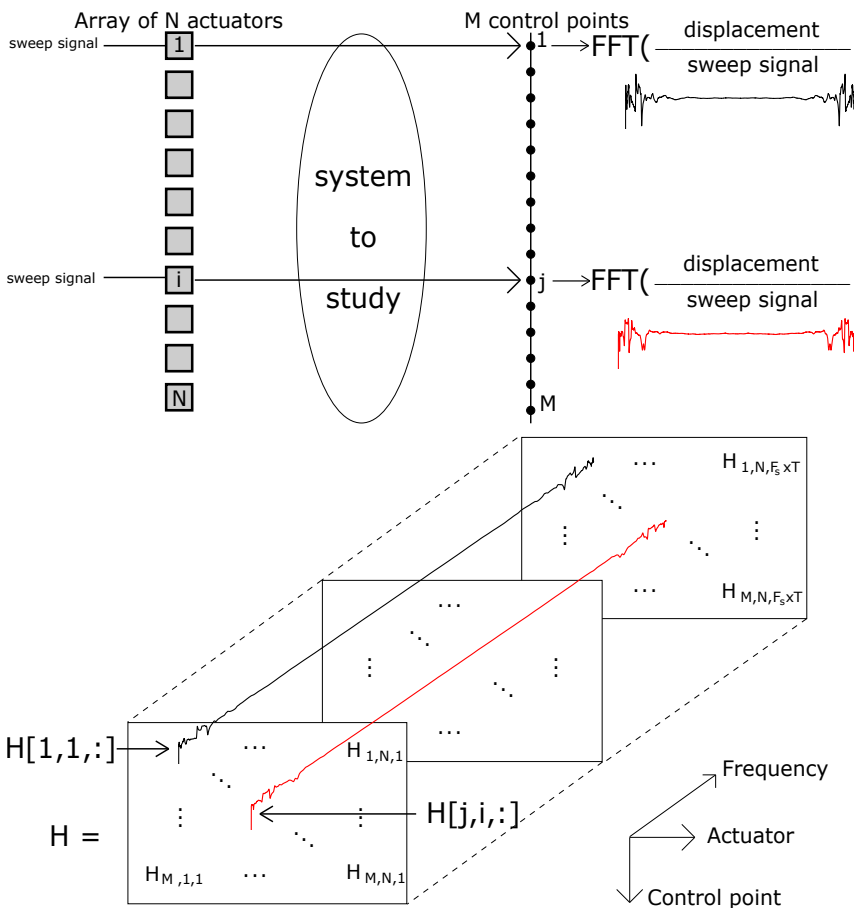

Fig. 2. Recuperation of the displacements on the different control points and calculation of the associated Fast Fourier Transform then put the different signals in the right place in order to build the matrix $\mathrm{H}$

tactile sensitivity range. The target amplitude was set to $1 \mu \mathrm{m}$ [18]. For all measured signals a correlation coefficient has been measured in order to verify the accuracy of the result. This coefficient is defined as follows:

$$
c_{u, v}=\sum_{t, j} u \cdot v / \sqrt{\sum_{t, j} u^{2} \cdot \sum_{t, j} v^{2}}
$$

where $u$ is the target signal and $v$ is the measured signal. In this expression, sums are made over both time $t$ and control points $j$. To have a good accuracy the correlation coefficient must be close to one.

No filtering or averaging were performed on the measured signals. In the first configuration, shown in Fig. 3, a first control point was activated with the $300 \mathrm{~Hz}$ burst while the second was activated with the $500 \mathrm{~Hz}$ burst. With the inverse filter approach, both phase and amplitude of the stimuli are controlled. We delayed the $500 \mathrm{~Hz}$ stimuli by $10 \mathrm{~ms}$ to demonstrate the ability to control precisely the time origin of each stimulus independently. Fig. 3 shows the control point positions as well as the target waveforms and the position of piezoelectric actuators.

The 14 actuators were used to control those two positions. Fig. 4 shows the signals for each of those actuator, in time domain, that were calculated based on the previously defined target displacements.

Finally, we measured the resulting displacement at the two selected control points. Those displacements, in Fig. 5, show a good agreement with the target displacement depicted in Fig. 3. Although small amplitude ripples are visible

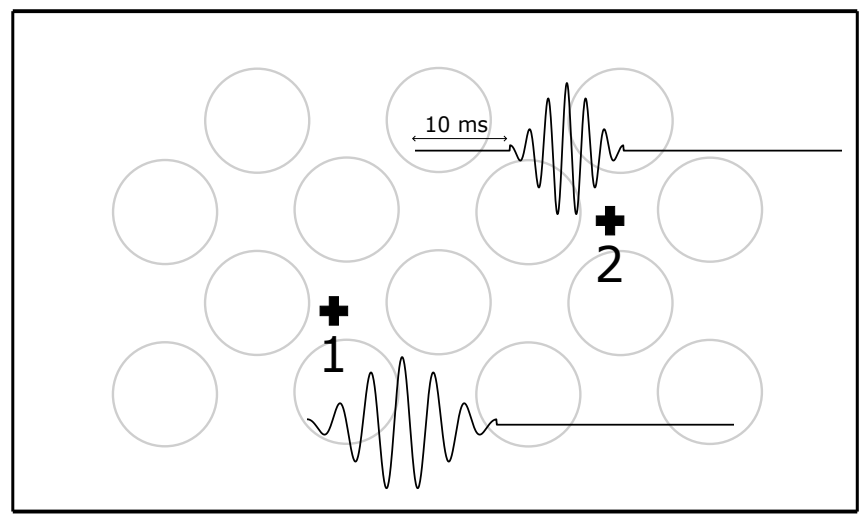

Fig. 3. Controlled output for the case one: first control point active with a $300 \mathrm{~Hz}$ burst and the second active with a $500 \mathrm{~Hz}$ burst with a delta of $10 \mathrm{~ms}$

before and after the stimuli, we observe that the waveform, amplitude and time location of the signals are very well preserved. This demonstrates the ability to control accurately the vibration by cancelling crosstalk and reverberation effects in the surface. It also illustrates the wideband aspect of this inverse filter approach that is capable of producing complex vibrotactile signals over the whole tactile sensitivity range.

We then investigated the ability to cancel vibrations at a location while delivering a $250 \mathrm{~Hz}$ stimulation at an other. This case is especially important in the perspective of multiuser interactions with a touch screen where stimuli delivered to one user must not be felt by an other. Those target vibrations are shown in Fig. 6.

Fig. 7 shows the measured displacements. This time again we observe a good agreement between the measured and target vibrations. The first control point is clearly passive while the second shows a well defined burst sine. Small amplitude residues are however visible on both signals with measurable amplitudes. On the passive, or muted, control point, the peak displacement reaches $0.1 \mu \mathrm{m}$, which is about three times lower than the detection threshold at the finger tip as measured by Verrillo [19]. Therefore, stronger stimuli could be provided to the active point while keeping the vibration unnoticeable at the muted position.

\section{Control on three positions}

We extended the number of control points to three in order to assess the stability of the algorithm and verify that sufficient vibration amplitudes could be reached even in a more constrained system. In a first experiment sine burst at $500 \mathrm{~Hz}$ and $700 \mathrm{~Hz}$ were produced at two positions while muting a third position. The control point positions and the waveforms are shown in Fig. 8. The results displayed in Fig. 9 shows that the waveform are preserved and that their amplitude reaches the nominal value of $1 \mu \mathrm{m}$.

The extension to three activated points, with target waveform at $300 \mathrm{~Hz}, 500 \mathrm{~Hz}$ and $700 \mathrm{~Hz}$, is shown in Fig. 10 . Fig. 11 shows a good agreement with the target vibrations, in terms of waveform, time delay and amplitude. 


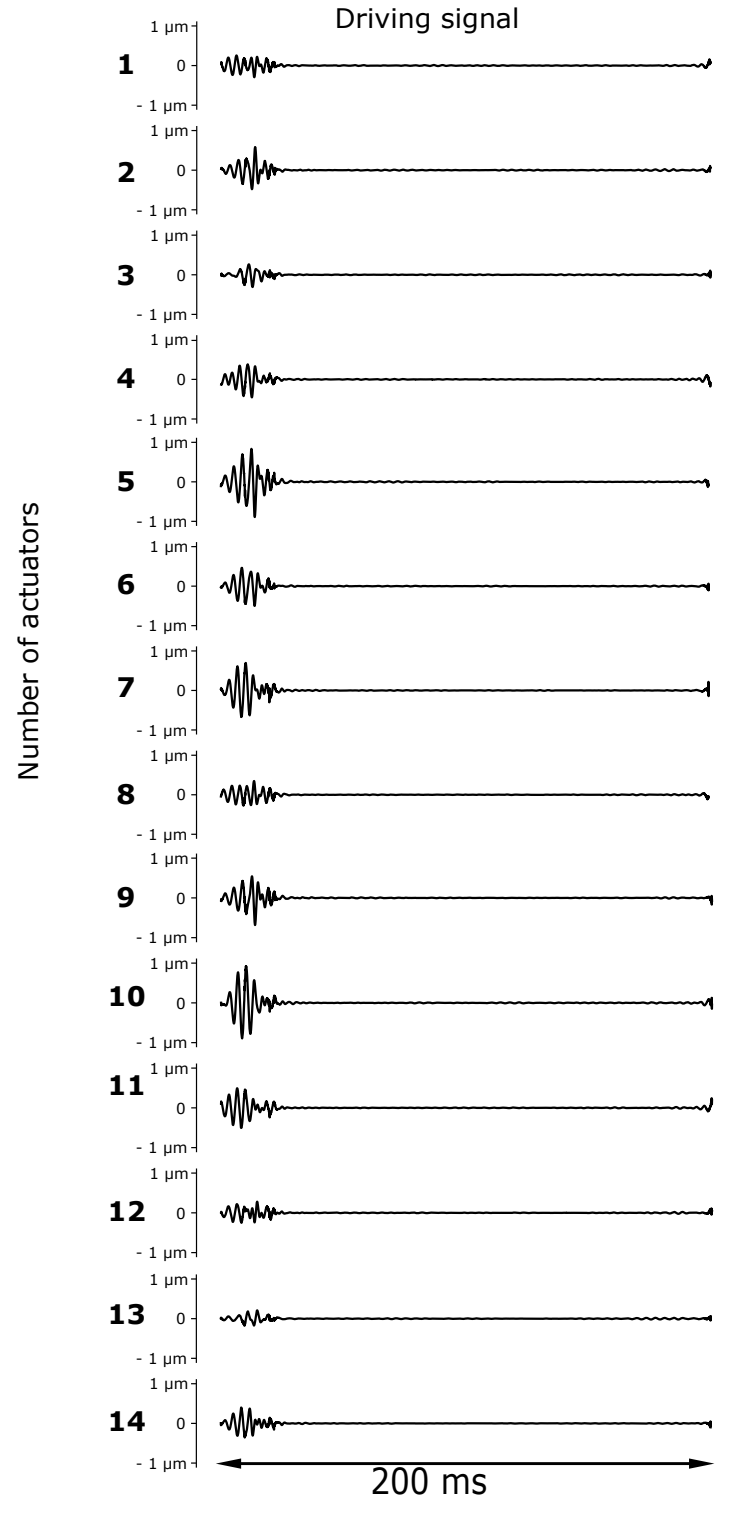

Fig. 4. Calculated signal $\mathbf{s}(t)$ for the case one using the spatio temporel filtering method: these signals will be sent to the actuators allowing us to create the displacement of the Figure 3

The technique therefore appears as robust with respect to the number of control points. Extending the number of control points indeed requires the same input data and signal processing. Identical behaviour are also obtained in terms of performance.

\section{E. Maximum amplitude}

Until now, the target vibrations were set to amplitudes of 1 $\mu \mathrm{m}$. In this experiment we amplified the signals to drive the piezoelectric actuators at the maximum amplitude allowed by the piezo drivers (200 Vpp). Points 1 and 3 were muted while a $500 \mathrm{~Hz}$ burst was produced at point 2 . A waveform with $3 \mu \mathrm{m}$ amplitude was thus produced, as shown in Fig. 12. This maximum amplitude is likely to variate as the position and number of control points vary. It however shows that waveforms of large amplitude can be produced with this

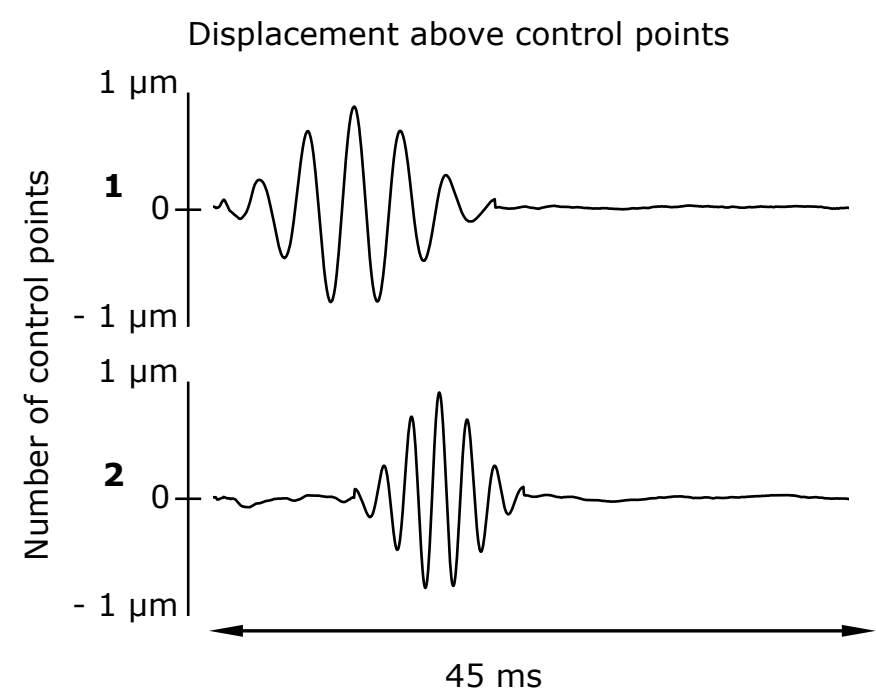

Fig. 5. Measurement at the two control points from the calculated signal $\mathbf{s}(t)$ (see Figure 4) used as input of the actuators. The correlation coefficient is 0.93 .

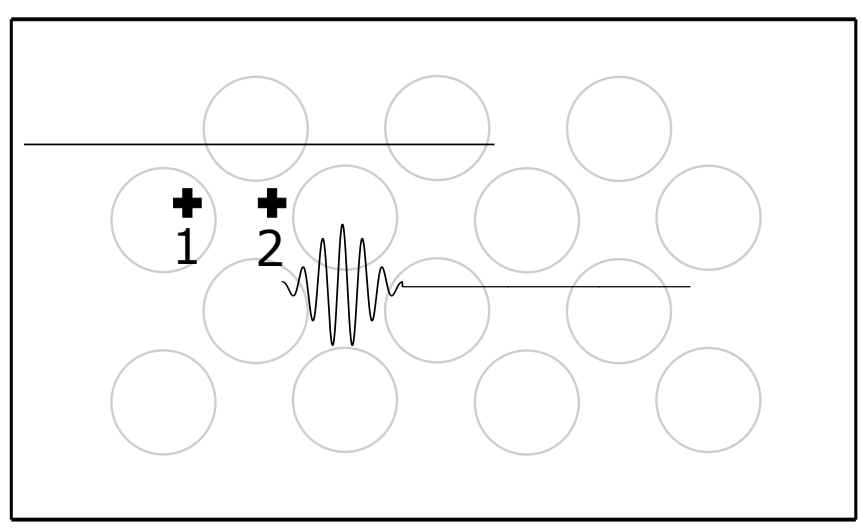

Fig. 6. Controlled output for the second case: first control point passive and the second active with a $250 \mathrm{~Hz}$ burst

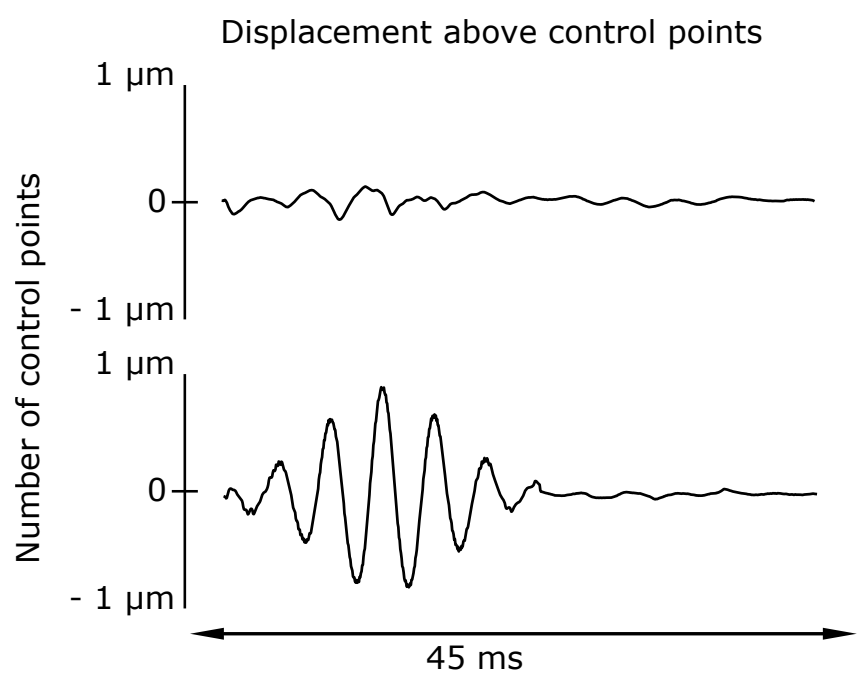

Fig. 7. Measure of displacements, above the actuators, of the case two. The correlation coefficient is 0.88 . 


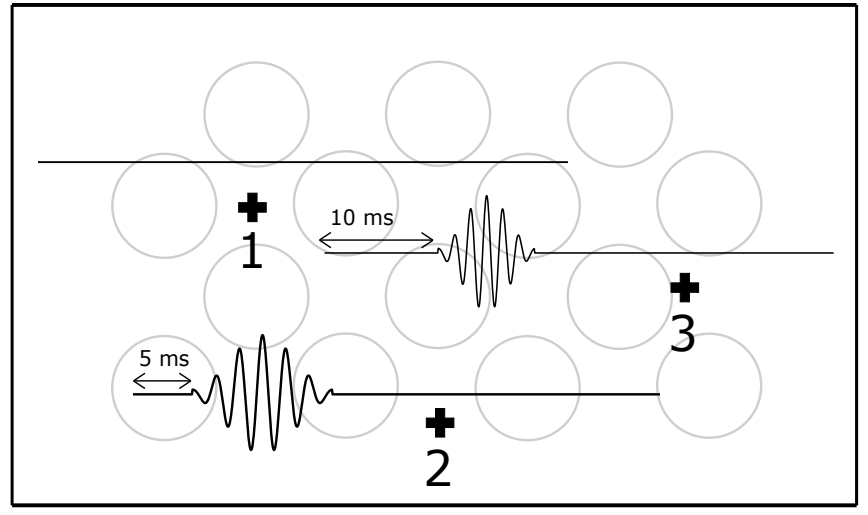

Fig. 8. Case three: first control point passive, second active with a $500 \mathrm{~Hz}$ burst and the third active with a $700 \mathrm{~Hz}$ burst

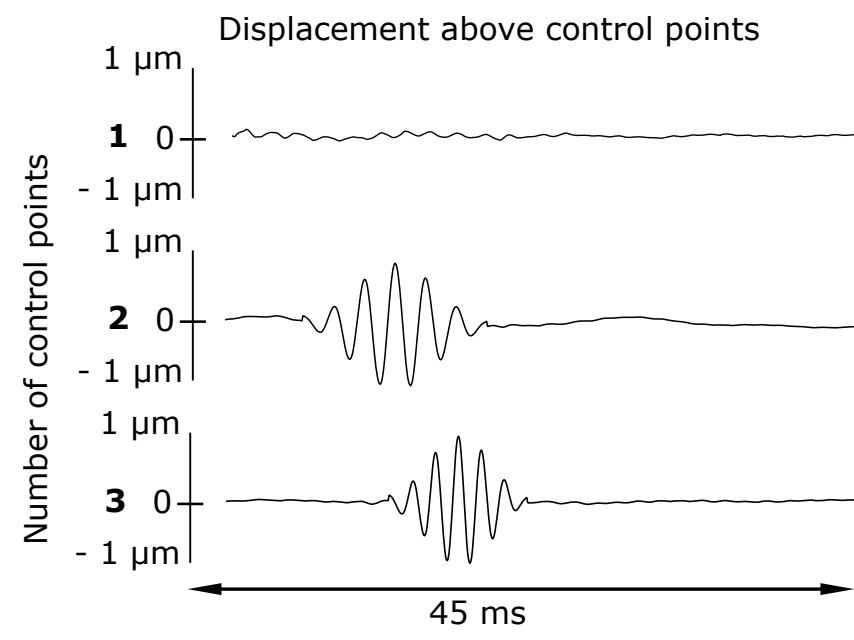

Fig. 9. Measure of displacements, above the actuators, of the case three. The correlation coefficient is 0.84 .

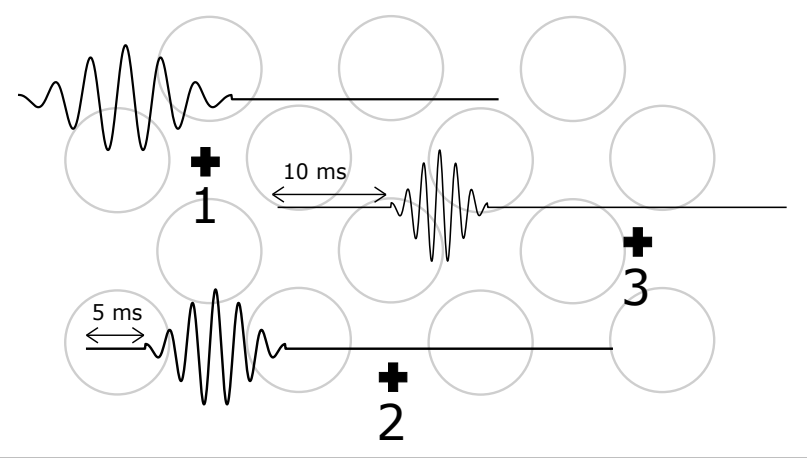

Fig. 10. Case four: first control point active with a $300 \mathrm{~Hz}$ burst, second active with a $500 \mathrm{~Hz}$ burst and the third active with a $700 \mathrm{~Hz}$ burst

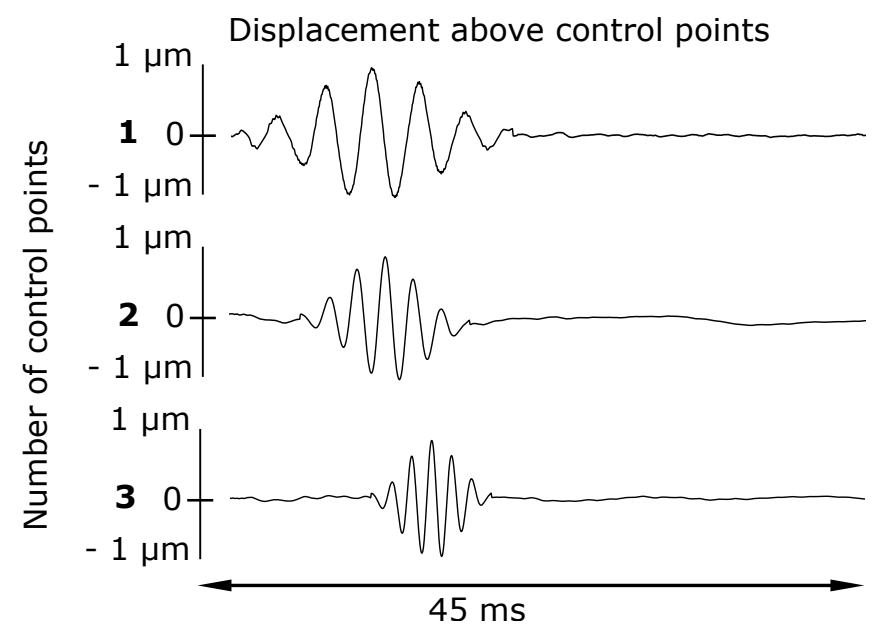

Fig. 11. Measure of displacements, above the actuators, of the case four The correlation coefficient is 0.91 .

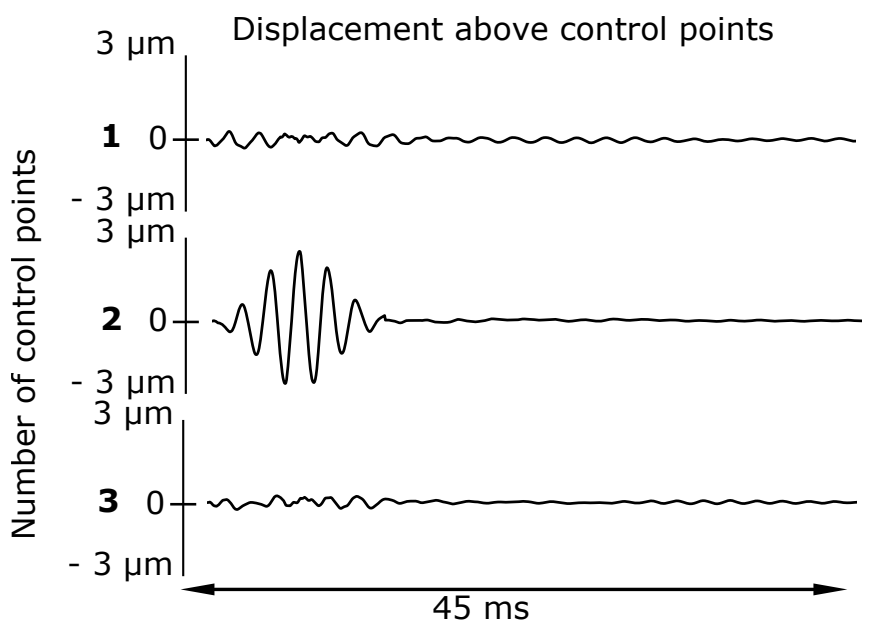

Fig. 12. A $500 \mathrm{~Hz}$ burst with $3 \mu \mathrm{m}$ of displacement. The correlation coefficient is 0.90 .

arrangement.

\section{INFLUENCE OF FINGERS CONTACT}

The Inverse Filter approach relies on a preliminary calibration and assumes that the transfer functions are not altered by the contact of fingers on the plate. Although the vibrations of mm thick plates are known to be relatively immune to finger contact, the inversion of ill-conditioned matrix are known to lead to unstable solutions that are sensitive to small variations. We therefore studied the influence of finger contact and applied force on the produced vibration. The configuration was the same as for the maximum amplitude study. Control points 1 and 3 were muted while point 2 was set with a $500 \mathrm{~Hz}$ burst with $1 \mu \mathrm{m}$ amplitude. A 24 years old man touched the plate above control point 2 while a vibrometer measured the plate displacement, as shown in Fig. 13.

Three measurements were made: without finger contact, with light touch and with a $5 \mathrm{~N}$ pressing force. The results are shown in Figure 14. Those results show that the signal 


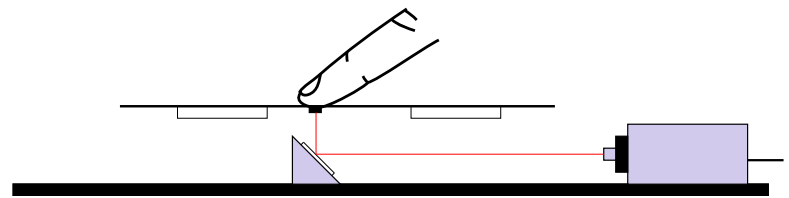

Fig. 13. Specific device in order to measure the displacement under the control points

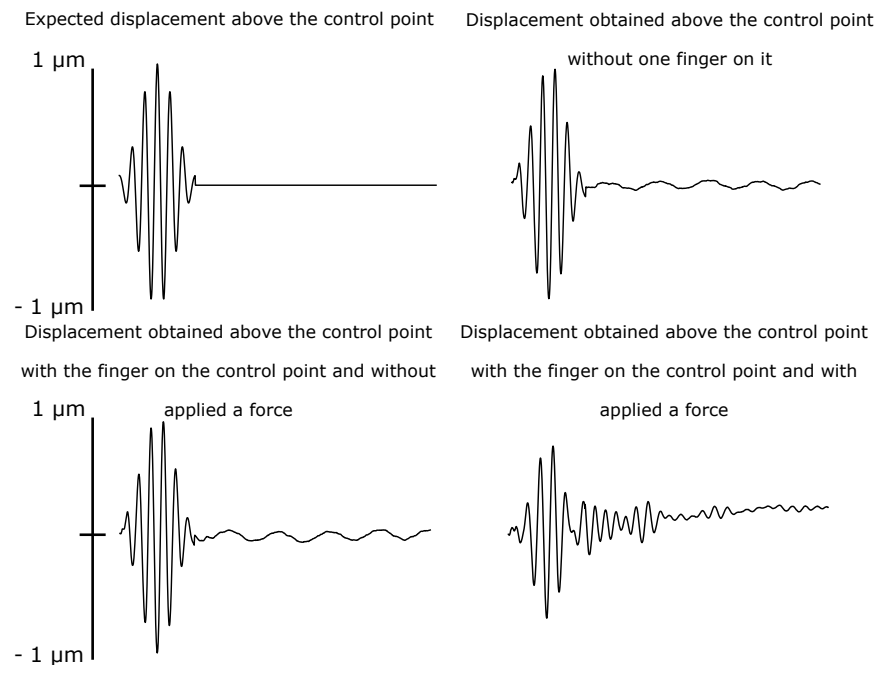

Fig. 14. Comparison between the different cases: 1) without the finger on the control point, 2) with the finger on the control point without applied force and 3) with the finger on the control point and with an applied force

waveform is not significantly altered by a light touch. The correlation coefficient between the signal without finger and with a light touch is indeed of 0.98 . This correlation however drops to 0.47 for the $5 \mathrm{~N}$ contact force. In this latter case the waveform is preserved but the amplitude decreases from $1 \mu \mathrm{m}$ to $0.7 \mu \mathrm{m}$. The signal also shows oscillations after the initial burst, which indicates that the inverse filter does not succeed any more at compensating signal reverberation in this condition of contact force. This is caused by a mismatch between the calibrated transfer functions and the actual ones. Further studies will help understanding this phenomenon whose origin could be the wave scattering by the finger, a variation of wave velocity due to the plate tension or a variation of boundary conditions.

\section{CONCLUSION}

We have shown in this article that the vibrations of a touch panel can be controlled independently at multiple locations by using a sparse array of piezoelectric actuators combined with the spatio temporal filtering method. With this approach, the waveform, amplitude and temporal location of stimuli are precisely controlled at arbitrary positions over the touch plate. Vibrotactile signals of various frequencies within the tactile sensitivity range were successfully reproduced simultaneously at up to three random positions over the plate, although this number of control point could be easily increased. Finger contact has shown to have little impact on the performances of this method for light touch conditions. For strong pressing forces however, the mismatch between the actual and calibrated systems decreases notably the quality of the delivered stimulus. Further research will be necessary to make this method more robust to contacts and variations of the surface mechanical properties and boundary conditions. The number, dimension and location of the actuators on the panel are expected to have a great impact on the performances of the method and will be investigated.

\section{REFERENCES}

[1] Byung-Kil Han, Kwangtaek Kim, Koji Yatani, and Hong Z Tan. Text entry performance evaluation of haptic soft qwerty keyboard on a tablet device. In Int. Conf. on Human Haptic Sensing and Touch Enabled Computer Applications, pages 325-332. Springer, 2014.

[2] Masaaki Fukumoto and Toshiaki Sugimura. Active click: tactile feedback for touch panels. In CHI'01 Extended Abstracts on Human Factors in Computing Systems, pages 121-122. ACM, 2001.

[3] Rock Leung, Karon MacLean, Martin Bue Bertelsen, and Mayukh Saubhasik. Evaluation of haptically augmented touchscreen gui elements under cognitive load. In Proc. of the ninth int. conf. on Multimodal interfaces - ICMI '07, page 374, Nagoya, Aichi, Japan, 2007. ACM Press.

[4] Matthew J. Pitts, Skrypchuk Lee, Tom Wellings, Alex Attridge, and Mark A. Williams. Evaluating user response to in-car haptic feedback touchscreens using the lane change test. Advances in HumanComputer Interaction, 2012.

[5] Johan Kildal. 3d-press: haptic illusion of compliance when pressing on a rigid surface. In Int. Conf. on Multimodal Int. and the Workshop on Machine Learning for Multimodal Interaction, page 21. ACM, 2010.

[6] Sunjun Kim and Geehyuk Lee. Haptic feedback design for a virtual button along force-displacement curves. In Proc. of the 26th annual ACM symposium on User interface software and technology, pages 91-96. ACM, 2013.

[7] Yon Visell, Bruno L. Giordano, Guillaume Millet, and Jeremy R. Cooperstock. Vibration Influences Haptic Perception of Surface Compliance During Walking. PLoS ONE, 6(3):e17697, March 2011.

[8] Charles Hudin, Jose Lozada, and Vincent Hayward. Localized Tactile Feedback on a Transparent Surface through Time-Reversal Wave Focusing. IEEE Transactions on Haptics, 8(2):188-198, April 2015.

[9] Takayuki Hoshi, Masafumi Takahashi, Takayuki Iwamoto, and Hiroyuki Shinoda. Noncontact Tactile Display Based on Radiation Pressure of Airborne Ultrasound. IEEE Transactions on Haptics, 3(3):155-165, July 2010.

[10] S. Ghenna, F. Giraud, C. Giraud-Audine, M. Amberg, and B. LemaireSemail. Preliminary design of a multi-touch ultrasonic tactile stimulator. In 2015 IEEE World Haptics Conference, pages 31-36, June 2015.

[11] K. Katumu and J. L. Gorlewicz. Using modal superposition for generating localized tactile effects on variable friction touchscreens. In 2016 IEEE Haptics Symposium, pages 211-216, April 2016.

[12] Jung-Han Woo and Jeong-Guon Ih. Vibration rendering on a thin plate with actuator array at the periphery. Journal of Sound and Vibration, 349:150-162, August 2015.

[13] M.R. Bai and Y.K. Tsai. Impact localization combined with haptic feedback for touch panel applications based on the time-reversal approach. J. Acoust. Soc. Am., 129:1297, 2011.

[14] S. Wöckel, U. Steinmann, and H. Arndt. Haptics by time reversal of elastic waves. In 2016 IEEE International Ultrasonics Symposium (IUS), pages 1-3, September 2016.

[15] Michael Tanter, J-F Aubry, J Gerber, J-L Thomas, and M Fink Optimal focusing by spatio-temporal inverse filter. i. basic principles. J. Acoust. Soc. Am., 110(1):37-47, 2001.

[16] Steven L Gay and Jacob Benesty. Acoustic signal processing for telecommunication, volume 551. Springer Science \& Business Media, 2012.

[17] Bruno Masiero, Janina Fels, and Michael Vorländer. Review of the crosstalk cancellation filter technique. Proc. of ICSA, page 112, 2011.

[18] Ronald T Verrillo, Anthony J Fraioli, and Robert L Smith. Sensation magnitude of vibrotactile stimuli. Perception \& Psychophysics, 6(6):366-372, 1969.

[19] Ronald T. Verrillo. Vibrotactile thresholds measured at the finger. Perception \& Psychophysics, 9(4):329-330, 1971. 